\title{
Star Formation Thresholds in Low Surface Brightness Dwarf Galaxies
}

\author{
Liese van Zee \\ National Radio Astronomy Observatory, Socorro, NM 87801
}

\begin{abstract}
The results of a comparative study of star formation thresholds in gas-rich, low surface brightness, dwarf galaxies are presented. Approximately half the galaxies in the study were "high $M_{H} / L_{B}$ " galaxies, which appear to have inefficient star formation properties. The comparison sample comprised of otherwise "normal" dwarf galaxies, with moderate current star formation rates. In all systems, sites of active star formation were associated with local peaks in the HI column density. For both types of galaxies, the azimuthally averaged gas column density is low. Similar to other LSB systems, the global gas densities are well below the critical threshold for star formation throughout the system. Thus, star formation is inhibited globally, but occurs locally in these gas-rich dwarf galaxies.
\end{abstract}

\section{Introduction}

One of the intriguing questions today is what mechanisms inhibit or assist star formation activity in low surface brightness (LSB) galaxies. Most LSB galaxies are gas-rich (e.g., Sprayberry et al. 1995), yet their low stellar densities imply that the star formation process has been inefficient in converting the available gas into stars. Several studies of the gas distribution in luminous LSB galaxies (e.g., van der Hulst et al. 1993; de Blok et al. 1996; Hunter et al. 1998) indicate that while these systems are gas-rich, their neutral gas surface densities fall well below the Toomre (1964) instability threshold throughout their optical disks. This is in direct contrast to the results for high surface brightness (HSB) galaxies, where the gas surface density exceeds the Toomre (1964) instability level in the inner disk, and star formation generally occurs in regions where the gas density exceeds $2 / 3$ of the Toomre criterion (Kennicutt 1989). Thus, the low gas surface density inherent in giant LSB galaxies may explain their low star formation rates and low stellar densities.

Several studies have recently applied the same star formation threshold arguments to low luminosity systems (e.g., Hunter \& Plummer 1996; van Zee et al. 1996; van Zee et al. 1997c; Hunter et al. 1998). One difficulty with this type of analysis for low mass systems is that their entire optical disks fall within the region of solid body rotation. Some debate has centered around whether the Toomre criterion is even valid in the limit of zero rotational shear; however, it should be valid for all types of single-fluid rotating disks since it is derived through perturbation analysis. Other threshold criteria, such as those 
derived from pressure balance (e.g., Elmegreen \& Parravano 1994) or those from two-fluid rotating disks (e.g., Hunter et al. 1998), have also been explored.

There are two additional caveats about the application of such criteria to observed gas distributions. First, most studies (with the notable exception of Kennicutt 1989) only include the distribution of the atomic gas since the molecular gas component is difficult to determine (or even detect!) (e.g., Wilson 1995; Verter \& Hodge 1995). Second, these criteria are applied to the azimuthally averaged gas surface density, which has the tendency to smooth over regions of high gas density/star formation activity. Thus, it is also important to investigate the correlation (or lack thereof) between the local gas density and sites of active star formation. Here, I summarize the results of a comparative study of global and local star formation thresholds in LSB and "normal" dwarf galaxies. Full details of the observations and results can be found in van Zee et al. (1997a,b,c).

\section{Sample Selection}

The primary sample of gas-rich low surface brightness dwarf galaxies were selected on the basis of unusually high values of $\mathrm{M}_{\mathrm{H}} / \mathrm{L}_{\mathrm{B}}(>5$, based on catalogued values). Although low surface brightness was not initially a selection criterion, the high $\mathrm{M}_{\mathrm{H}} / \mathrm{L}_{\mathrm{B}}$ systems tend to be low surface brightness objects. In these systems, a significant fraction of their total luminosity comes from regions outside of $D_{25}$ and thus their catalogued magnitudes were severe underestimates of their true luminosity. As discussed by Patterson \& Thuan (1996) and van Zee et al. (1997a), the catalogued magnitudes underestimate the true magnitudes by $\sim 1.5^{m}$ for low surface brightness dwarf galaxies. Thus, systems which appear in the catalogues with extremely high $\mathrm{M}_{\mathrm{H}} / \mathrm{L}_{\mathrm{B}}$ are typically low surface brightness systems for which the optical magnitudes have been incorrectly estimated. However, even with revised luminosities derived from deep optical imaging, these systems still have values of $M_{H} / L_{B}$ which are typically factors of two or more higher than normal for dwarf galaxies.

A comparison sample of "normal" dwarf galaxies were also observed. These systems have similar global properties to the LSB dwarfs, but have values of $\mathrm{M}_{\mathrm{H}} / \mathrm{L}_{\mathrm{B}} \sim 1$. In particular, these objects are not blue compact dwarf galaxies. Rather, they are low luminosity dwarf galaxies which have approximately the same linear optical sizes as the LSB systems but slightly higher central surface brightnesses and star formation rates. Note that both the LSB and the normal dwarf galaxies in this sample are rotation dominated systems, with typical maximum rotation velocities of $40-100 \mathrm{~km} \mathrm{~s}^{-1}$. Finally, both the normal and LSB dwarf samples were selected to be isolated systems, based on inspection of the Palomar Observatory Sky Survey (POSS) prints.

The current star formation rates for both the LSB and normal dwarfs are quite low and span a wide range, from 0.0015 to $0.35 \mathrm{M}_{\odot} \mathrm{yr}^{-1}$. Qualitatively, the $\mathrm{H} \alpha$ emission from the normal dwarfs tends to be concentrated in discrete knots near the center of the system, while several of the LSB dwarfs have a significant contribution of diffuse $\mathrm{H} \alpha$ emission. An example of the extremely low star formation rates found in the LSB dwarf galaxies is shown in Figure 1b. 


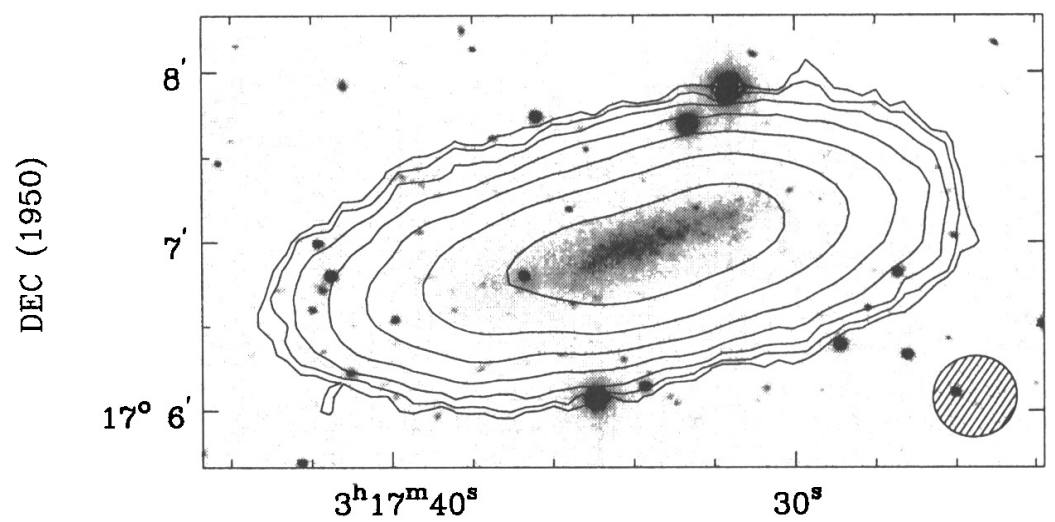

RA (1950)

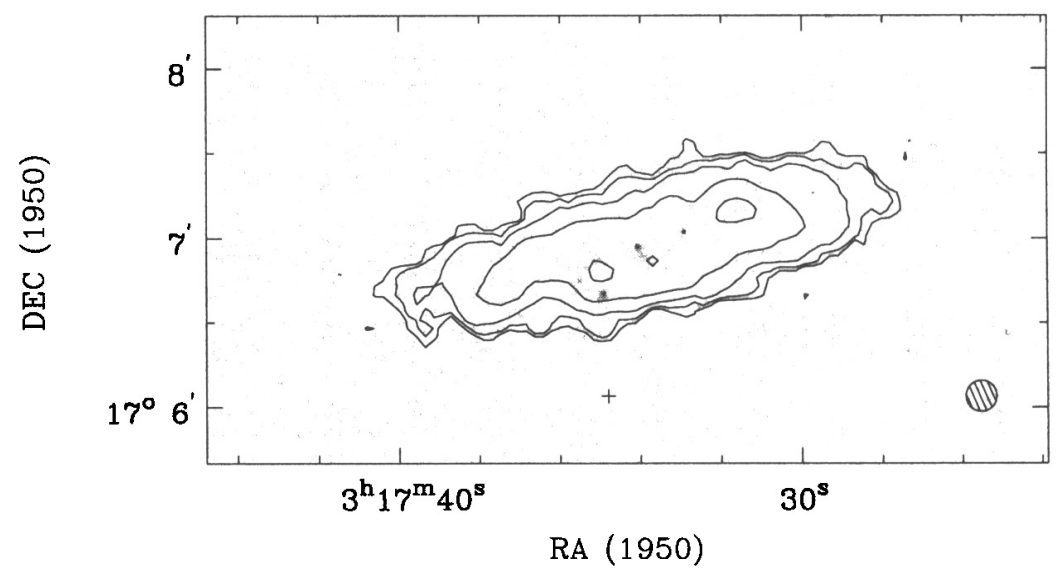

Figure 1. The HI column density distribution of UGC 2684, a low surface brightness dwarf galaxy. The upper panel shows a B-band image of UGC 2684 with a low resolution $\left(23.7^{\prime \prime} \times 22.0^{\prime \prime}\right)$ HI map superposed. The HI contours are logarithmic, with the lowest and highest contours corresponding to densities of $2.0 \times 10^{19}$ and $1.3 \times 10^{21}$ atoms $\mathrm{cm}^{-2}$, respectively. The lower panel shows a continuum subtracted $\mathrm{H} \alpha$ image of UGC 2684 with a higher resolution $\left(11.1^{\prime \prime} \times 11.1^{\prime \prime}\right)$ HI map superposed. The HI contours are logarithmic, with the lowest and highest contours corresponding to densities of $1.6 \times 10^{20}$ and $2.6 \times 10^{21}$ atoms $\mathrm{cm}^{-2}$, respectively. Note the coincidence between sites of active star formation and local peaks in the HI surface density. 


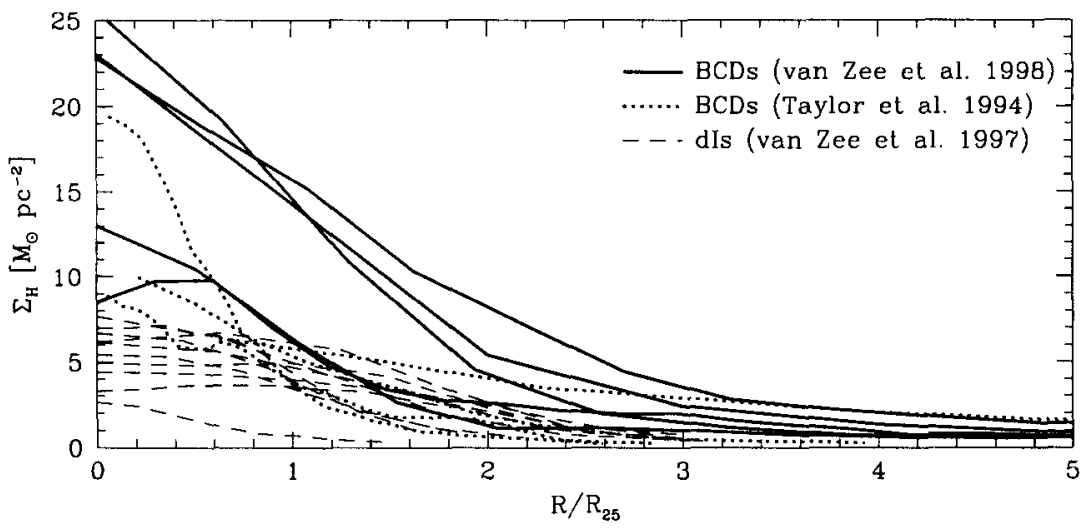

Figure 2. Radial HI surface densities for BCDs and dIs. The BCDs (Taylor et al. 1994; van Zee et al. 1998) have much higher central surface densities than comparable dIs (van Zee et al. 1997c).

\section{Star Formation Thresholds}

Azimuthally averaged gas surface density profiles of the normal and LSB dwarf galaxies are shown in Figure 2. Also included in this figure are the results of HI studies of star-bursting blue compact dwarf (BCD) galaxies (Taylor et al. 1994; van Zee et al. 1998). A sharp contrast is found between the more quiescent dwarf irregular galaxies (both LSB and "normal") and the star-bursting BCD galaxies. Not only are the HI distributions of the BCDs centrally concentrated, their central surface densities are significantly higher than those of the dIs. Thus, it appears that star-bursting BCDs have inherently different gas mass distributions than dIs, which may be a clue to the origin of strong star formation activity in BCDs.

The ratio between the azimuthally averaged gas surface density and the Toomre (1964) instability criterion for the LSB and normal dwarf galaxy samples are shown in Figure 3 (upper and lower panels, respectively). Of particular interest is the fact that none of the LSB dwarf galaxies have gas densities which exceed the Toomre instability level. Again, this is in direct contrast with results for blue compact dwarf galaxies, where the Toomre criterion is exceeded throughout the star forming regions (Taylor et al. 1994). However, little difference is seen when the LSB and normal dwarf galaxies (objects with similar scale lengths, but slightly higher surface brightnesses) are compared. Similar results were found by Hunter et al. (1998) in their study of gas density thresholds for dwarf galaxies. Thus, it appears that star formation is inhibited for both the LSB and normal dwarf galaxies; in both types of systems, the global gas density appears to be too low to support percolation of star formation.

So, how do these systems form stars at all if their gas density is too low? One important consideration is the fact that star formation is a local process in dwarf irregular systems. Unlike the massive spiral galaxies (both HSB and 

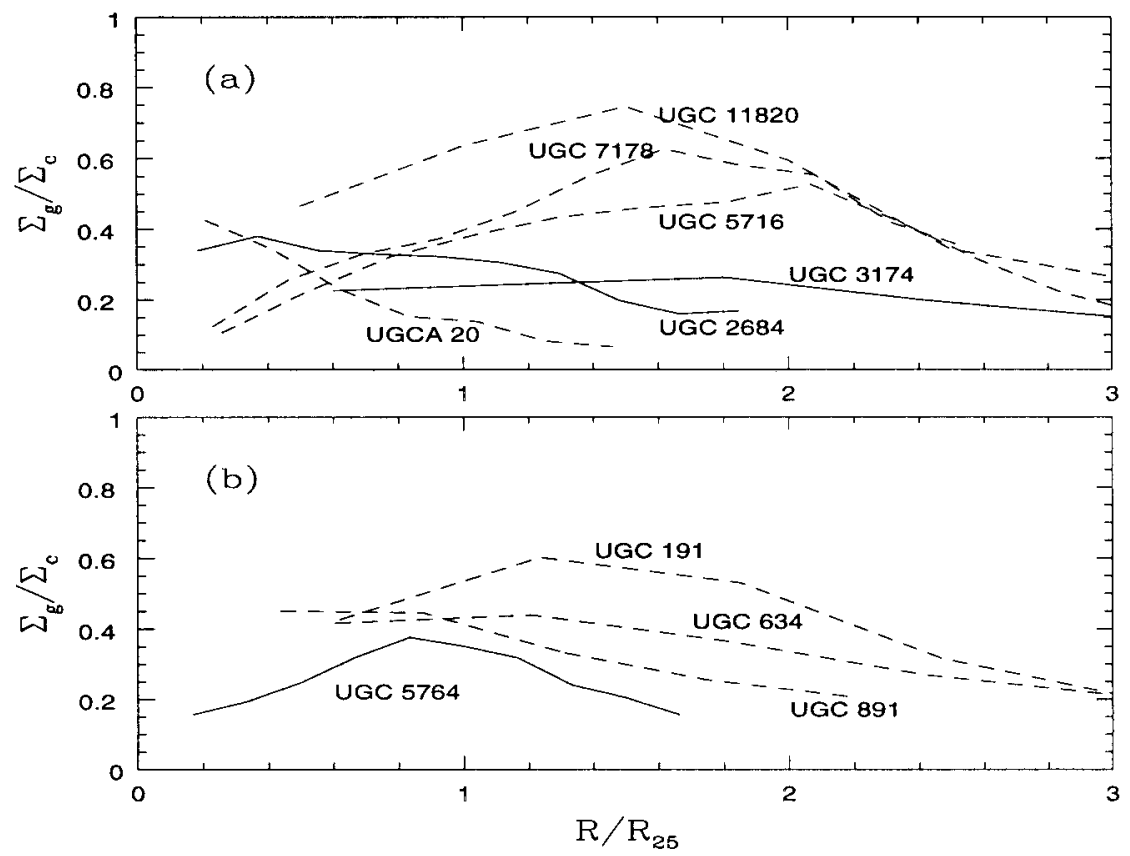

Figure 3. The ratio between the observed neutral hydrogen surface density and the Toomre instability threshold density. (a) The low surface brightness dwarf galaxies. (b) The "normal" dwarfs. The dashed lines denote galaxies with optical scale lengths greater than $1.5 \mathrm{kpc}$. Note that all of the galaxies have gas densities lower than the predicted instability threshold, but that the intrinsically larger systems do have slightly higher surface densities.

LSB) where spiral density waves introduce global patterns in the star formation activity, the onset of star formation is primarily stochastic in low mass systems. Thus, the azimuthal averages shown in Figures 2 and 3 are not representative of the local gas density in regions of active star formation. As seen in Figure $1 \mathrm{~b}$, there is a good correlation between sites of star formation activity and high column density regions on the local level. Thus, while star formation may be inhibited globally in both the LSB and normal dwarf galaxies, it is permitted locally.

\section{Are Low Surface Brightness Dwarf Galaxies Young?}

The high ratios of $M_{H} / L_{B}$ found for the LSB dwarf galaxies suggest that much of the gas in these systems has not yet been processed into stars. Two possible explanations for this are that either the time averaged star formation rate is extremely low in these objects, or that star formation has been initiated recently. Under the first hypothesis, inefficient star formation would slowly convert gas into stars over a Hubble time, resulting in a mixed stellar population at the 

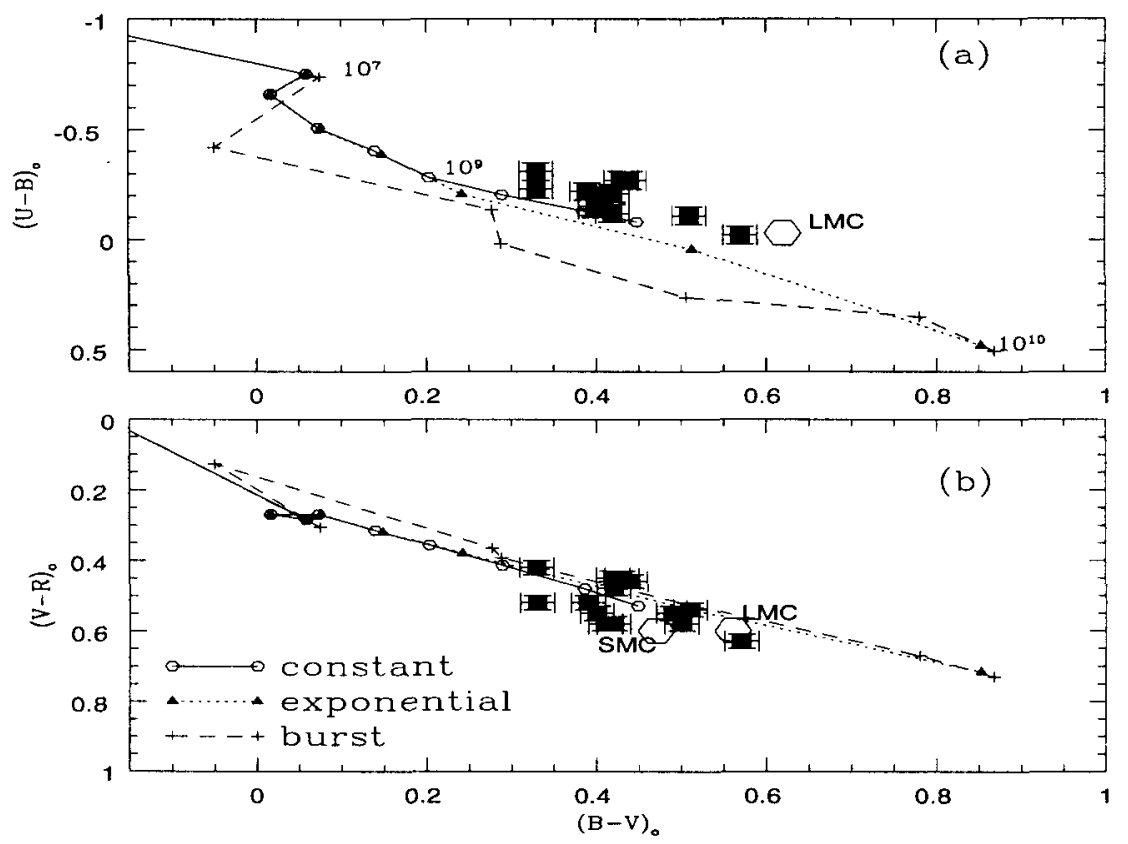

Figure 4. UBV and BVR color-color diagrams for the low surface brightness dwarf galaxies. The color evolution for 3 different star formation rate laws are shown (Bruzual \& Charlot 1993). The colors for a galaxy with a Salpeter IMF and constant star formation rate is shown with a solid line. The evolution of a $10^{7}$ year burst is shown with a dashed line. An exponentially decreasing star formation rate with an e-folding time of 1 Gyr is shown with a short dashed line. The time range for each model is marked from $10^{7}$ to $10^{10}$ years every 0.5 dex.

present epoch. Under the second hypothesis, the recent onset of star formation would result in a predominately young stellar population at the present epoch. Further, in this scenario, the recent star formation rate would have to be relatively high to have generated the observed luminosities. Thus, the observed colors and current star formation rates could distinguish between these two possible evolutionary histories.

The results of UBVR broad band imaging of the low surface brightness dwarf galaxies are shown in Figure 4 (van Zee et al. 1997b). Also plotted in Figure 4 are the color evolution tracks of Bruzual \& Charlot (1993). These models assume solar metallicity, which is clearly an incorrect assumption for most of these systems. Thus, the ages derived below are lower limits due to the unknown level of the line blanketing effect.

Age determinations from color evolution models require two major assumptions: (1) the IMF, and (2) the star formation rate history. We have assumed an IMF of Salpeter form and experimented with three different star formation rate histories: (1) the evolution of a single burst of $10^{7}$ year duration, (2) an 
exponentially decreasing star formation rate with an e-folding time of $1 \mathrm{Gyr}$, and (3) a constant star formation rate.

In the BVR plane, all three star formation rate histories are degenerate. In this plane it is impossible to distinguish between an aging burst population of 1 Gyr, a 3 Gyr old system with an exponentially decreasing star formation rate, and a 15 Gyr old system with a constant star formation rate.

In the UBV plane, an aging burst population is too red to fit any of the observed colors. This is not unexpected since these systems are fairly large and isolated; it is thus unlikely that the entire system has undergone a simultaneous burst of star formation. The exponentially decreasing star formation rate and constant star formation rates come close to fitting the observed colors (the remaining offset is probably due to using solar metallicity models for subsolar metallicity systems). Both of these star formation rate histories indicate that the dominant stellar populations of the LSB dwarf galaxies were formed within the last 2-4 Gyr. Furthermore, it is not unreasonable to assume that older stellar populations are also present in the LSB dwarf galaxies.

Thus, based on the observed colors and current star formation rates, it appears that one reasonable explanation for the observed enhanced $M_{H} / L_{B}$ is that the star formation rate has been extremely low in these objects. It is highly unlikely that these objects are truly "young" systems undergoing their first episode of star formation. Rather, it appears that the star formation process is simply inefficient.

\section{Conclusions}

In conclusion, we find that the global gas surface density in both the LSB and the normal dwarf galaxies is significantly below the critical surface density at the present epoch. In fact, the two samples appear remarkably similar in HI, despite differences in their optical properties (surface brightness, $\mathrm{M}_{\mathrm{H}} / \mathrm{L}_{\mathrm{B}}$, etc.). Further, in both types of galaxies the sites of active star formation are correlated with local peaks in the gas surface density, which approach the instability criterion. Thus, star formation appears to be inhibited globally, but permitted locally in low mass galaxies.

The initiation of current star formation in these objects is probably due to self-regulating feedback, where local regions approach or exceed the critical density to form new HI clouds. These clouds collapse to form stars, once again decreasing the local surface density. Propagation of star formation through the disk may occur if the neighboring gas is compressed by the subsequent evolution of massive stars (e.g., stellar winds, ionizing flux, and supernovae explosions). Clearly, it is the latter step which inhibits star formation if the global surface density is too low. Thus, one possible explanation for the slightly higher surface brightness of the normal dwarfs is the initial gas density distribution. Perhaps the normal dwarfs started with a higher gas density distribution, but then processed their "excess" gas into stars until star formation became too inefficient (i.e., the present epoch). If so, this could also explain why the normal dwarfs have a lower ratio of $\mathrm{M}_{\mathrm{H}} / \mathrm{L}_{\mathrm{B}}$ than the LSB dwarf galaxies, since they have converted a larger fraction of their initial gas into stars. 
Finally, despite their blue colors and abundant supply of relatively unprocess gas, the low surface brightness dwarf galaxies do not appear to be young systems. Rather, their dominant stellar populations are 2-4 Gyr old, and star formation has probably been underway for a large fraction of a Hubble time.

Acknowledgments. I thank my collaborators, Martha Haynes, John Salzer and Adrick Broeils, for many useful conversations. The National Radio Astronomy Observatory is a facility of the National Science Foundation, operated under a cooperative agreement by Associated Universities Inc.

\section{References}

Bruzual, G., \& Charlot, S. 1993, ApJ, 405, 538

de Blok, W. J. G., McGaugh, S. S., \& van der Hulst, J. M. 1996, MNRAS, 283, 18

Elmegreen, B. G., \& Parravano, A. 1994, ApJ, 435, L121

Hunter, D. A., Elmegreen, B. G., \& Baker, A. L. 1998, ApJ, 493, 595

Hunter, D. A., \& Plummer, J. D. 1996, ApJ, 462, 732

Kennicutt, R. C. 1989, ApJ, 344, 685

Patterson, R. J., \& Thuan, T. X. 1996, ApJS, 107, 103

Sprayberry, D., Bernstein, G. M., \& Impey, C. D. 1995, ApJ, 438, 72

Taylor, C. L., Brinks, E., Pogge, R. W., \& Skillman, E. D. 1994, AJ, 107, 971

Toomre, A. 1964, ApJ, 139, 1217

van der Hulst, J. M., Skillman, E. D., Smith, T. R., Bothun, G. D., McGaugh,

S. S, \& de Blok, W. J. G. 1993, AJ, 106, 548

van Zee, L., Haynes, M. P., \& Salzer, J. J. 1997a, AJ, 114, 2479

van Zee, L., Haynes, M. P., \& Salzer, J. J. 1997b, AJ, 114, 2497

van Zee, L., Haynes, M. P., Salzer, J. J., \& Broeils, A. H. 1996, AJ, 112, 129

van Zee, L., Haynes, M. P., Salzer, J. J., \& Broeils, A. H. 1997c, AJ, 113, 1618

van Zee, L., Skillman, E. D., \& Salzer, J. J. 1998, AJ, 116, 1186

Verter, F., \& Hodge, P. W. 1995, ApJ, 446, 616

Wilson, C. D. 1995, ApJ, 448, L97 